\title{
Incidence of pneumonitis/interstitial lung disease induced by HER2-targeting therapy for HER2-positive metastatic breast cancer
}

\author{
Michelle D. Hackshaw ${ }^{1}$ (1) $\cdot$ Heather E. Danysh ${ }^{2} \cdot$ Jasmeet Singh $^{1} \cdot$ Mary E. Ritchey ${ }^{3} \cdot$ Amy Ladner $^{3} \cdot$ Corina Taitt $^{1}$. \\ D. Ross Camidge ${ }^{4} \cdot$ Hiroji Iwata $^{5} \cdot$ Charles A. Powell $^{6}$
}

Received: 18 May 2020 / Accepted: 13 June 2020 / Published online: 26 June 2020

(c) The Author(s) 2020

\begin{abstract}
Purpose Anti-human epidermal growth factor receptor 2 (HER2) therapies are associated with interstitial lung disease (ILD), also referred to as pneumonitis. In this literature review, we describe the incidence of ILD among patients with HER2-positive metastatic breast cancer $(\mathrm{MBC})$ receiving anti-HER2 therapies, and we describe existing recommendations for monitoring and managing drug-induced ILD among these patients.

Methods We searched PubMed and Embase to identify clinical trials and postmarket observational studies that investigated anti-HER2 therapies for HER2-positive MBC, reported on ILD, and were published during January 1, 2009 to July 15, 2019. Articles were screened by two researchers; data were extracted from the full-text articles.

Results The 18 articles selected for this review assessed 9,886 patients who received trastuzumab ( 8 articles), lapatinib (4 articles), trastuzumab emtansine ( 3 articles), trastuzumab deruxtecan ( 2 articles), or trastuzumab duocarmazine ( 1 article). The overall incidence of all-grade ILD was $2.4 \%(n=234)$, with $66.7 \%(n=156)$ occurring as grade $1-2$ events, $0.5 \%$ grade 3-4 ( $n=54$; incidence), and $0.2 \%$ grade 5 ( $n=16$; incidence). The highest ILD incidence $(21.4 \%)$ was among patients receiving trastuzumab combined with everolimus and paclitaxel. Ten studies indicated that ILD events were managed via dose interruption, dose reduction, or treatment discontinuation; two studies included detailed guidelines on managing druginduced ILD.

Conclusions ILD is a well-described adverse drug reaction associated with several anti-HER2 drugs. Published ILD management guidelines are available for few anti-HER2 treatment regimens; however, guidance for monitoring for anti-HER2 drug-induced ILD is lacking.
\end{abstract}

Keywords Metastatic breast cancer · HER2 positive - Interstitial lung disease · HER2-targeting therapy · Trastuzumab · Lapatinib · Trastuzumab emtansine $\cdot$ Trastuzumab deruxtecan $\cdot$ Trastuzumab duocarmazine

\section{Introduction}

Electronic supplementary material The online version of this article (https://doi.org/10.1007/s10549-020-05754-8) contains supplementary material, which is available to authorized users.

Michelle D. Hackshaw

mhackshawel@dsi.com

1 Daiichi Sankyo, Inc., 211 Mount Airy, 1A-453, Basking Ridge, NJ 07920, USA

2 RTI Health Solutions, Waltham, MA, USA

3 RTI Health Solutions, Research Triangle Park, NC, USA

4 University Colorado, Aurora, CO, USA

5 Aichi Cancer Center, Nagoya, Japan

6 Mount Sinai Hospital, New York, NY, USA
Approximately $15-20 \%$ of patients with breast cancer have tumors that overexpress human epidermal growth factor receptor 2 (HER2), which are associated with an aggressive clinical phenotype and poor prognosis $[1,2]$. The development and clinical integration of anti-HER2 therapies, including trastuzumab, pertuzumab, lapatinib, neratinib, and trastuzumab emtansine (T-DM1), has resulted in extended survival in patients with HER2-positive breast cancer [3-7]. Several anti-HER2 therapies, as well as other anticancer agents (e.g., cyclin-dependent kinase-4/-6 inhibitors, immune checkpoint inhibitors, mammalian target of rapamycin [mTOR] inhibitors), have been linked to an increased risk of drug-induced interstitial lung disease (ILD) 
[3, 4, 7-10]. Drug-induced ILD encompasses a group of serious, and sometimes life-threatening pulmonary conditions characterized by fibrosis and inflammation of the lung interstitium $[11,12]$.

Corticosteroids or oral prednisone are frequently used pharmacologic therapies for ILD that may effectively slow or reverse ILD disease progression [12]. While guidance is available on the diagnosis and treatment of drug-induced ILD [13], there is limited guidance on disease monitoring and management for patients with ILD induced by HER2targeted therapies. This may be due to variability in the terminology used to describe ILD in the literature, as well as limited provider experience in routinely diagnosing and managing ILD [12, 14].

The objective of this review is to describe the incidence and severity of drug-induced ILD across currently approved and investigational anti-HER2 therapies in female patients with metastatic breast cancer (MBC), and describe recommendations for monitoring and management of anti-HER2 drug-induced ILD among MBC patients.

\section{Methods}

PubMed and Embase databases were searched for phase 2, 3 , and 4 clinical studies that assessed current and investigational therapies for HER2-positive MBC. The predefined search terms included combinations of free text and Medical Subject Headings (MeSH). All databases were searched for English-language articles only, during the period of January 1, 2009, through July 15, 2019, and with no geographic restrictions imposed. Bibliographies of systematic literature reviews (SLRs) and recent articles published after the database search time window were examined to identify additional relevant publications.

Article screening and selection were conducted in two phases. In the first phase, titles and abstracts of articles identified from the electronic databases were reviewed by one researcher for eligibility according to the inclusion and exclusion criteria, which are outlined in Table S-1, Supplementary Material, including the terms used to describe ILD (i.e., pulmonary fibrosis, pneumonitis, organizing pneumonia, acute interstitial pneumonitis, diffuse parenchymal lung disease, pulmonary eosinophilia, ILD). In the second phase, the full text of the articles selected in the first phase was obtained and reviewed for eligibility by a second researcher using the same inclusion and exclusion criteria. Uncertainty about the inclusion of articles was resolved by discussion and consensus of two researchers. Information was extracted from full-text publications using a data extraction template with prespecified fields. Quality control procedures included verification of all extracted data with original sources by a researcher who did not perform the primary data extraction.

\section{Results}

Of the 240 studies identified through database searching, 158 titles and abstracts were identified in the PubMed and Embase databases after removing duplicate articles, as well as eight additional articles identified through the SLR bibliography search (Fig. 1). Of the 166 total articles that were reviewed in the first screening phase, 76 were eligible for a full-text review based on the inclusion and exclusion criteria. During the full-text review, a total of 60 articles were excluded, with the majority $(n=45$ [75.0\%]) excluded because they did not report on ILD. Fifteen articles from the electronic database search and one from the SLR bibliography search were eligible for inclusion in the review. Two additional relevant studies (one for trastuzumab deruxtecan [T-DXd] [15] and one for trastuzumab duocarmazine [16]) were identified via manual searches, yielding a total of 18 articles that were included in the review.

The study and patient characteristics for the 18 articles in the review are presented in Table 1. Studies reported at least $99 \%$ enrollment of female patients, except for one study that reported enrolling $77 \%$ and $82 \%$ female patients in two study cohorts [16]. Age (median) at enrollment ranged from 47 to 57 years [15-31]. In 12 studies, 54\% to $100 \%$ of patients were white [15-17, 19-21, 23-26, 29, 31]. A majority of enrolled patients were Asian in two studies (54\% [27] and 100\% [30]). Two studies reported nearly equivalent proportions of white and Asian patients $[22,32]$. Most studies $(n=11)$ were conducted in an international setting, enrolling patients in multiple countries and continents. Three studies were conducted solely in the United States (US) [17, 24, 31], while others enrolled patients in both the US and Japan [27], Europe [16], France [18], or China [30].

The reported proportion of patients with positive hormone receptor (i.e., estrogen receptor and/or progesterone receptor) status ranged from 42 to $70 \%$. Nearly all patients had an Eastern Cooperative Oncology Group (ECOG)/World Health Organization performance status of 0 or 1 (at least $92 \%$ in each treatment group); however, five studies enrolled a minimal number of patients with an ECOG performance status of 2, ranging from less than $1 \%$ to $8 \%$ of patients $[15,19,24,26,29]$. The shortest time (median) since breast cancer diagnosis was 22 months [32] and the longest was 71.5 months (among patients negative for programmed cell death-1 ligand-1 [PD-L1]) [28]. All but one study [25] included patients who had received previous breast cancer treatment. Among the six studies that reported on radiotherapy history [18, 19, 22, 25, 27, $31]$, the proportion of patients with previous radiotherapy ranged from $30 \%$ [18] to $100 \%$ [19]. The reported previous 


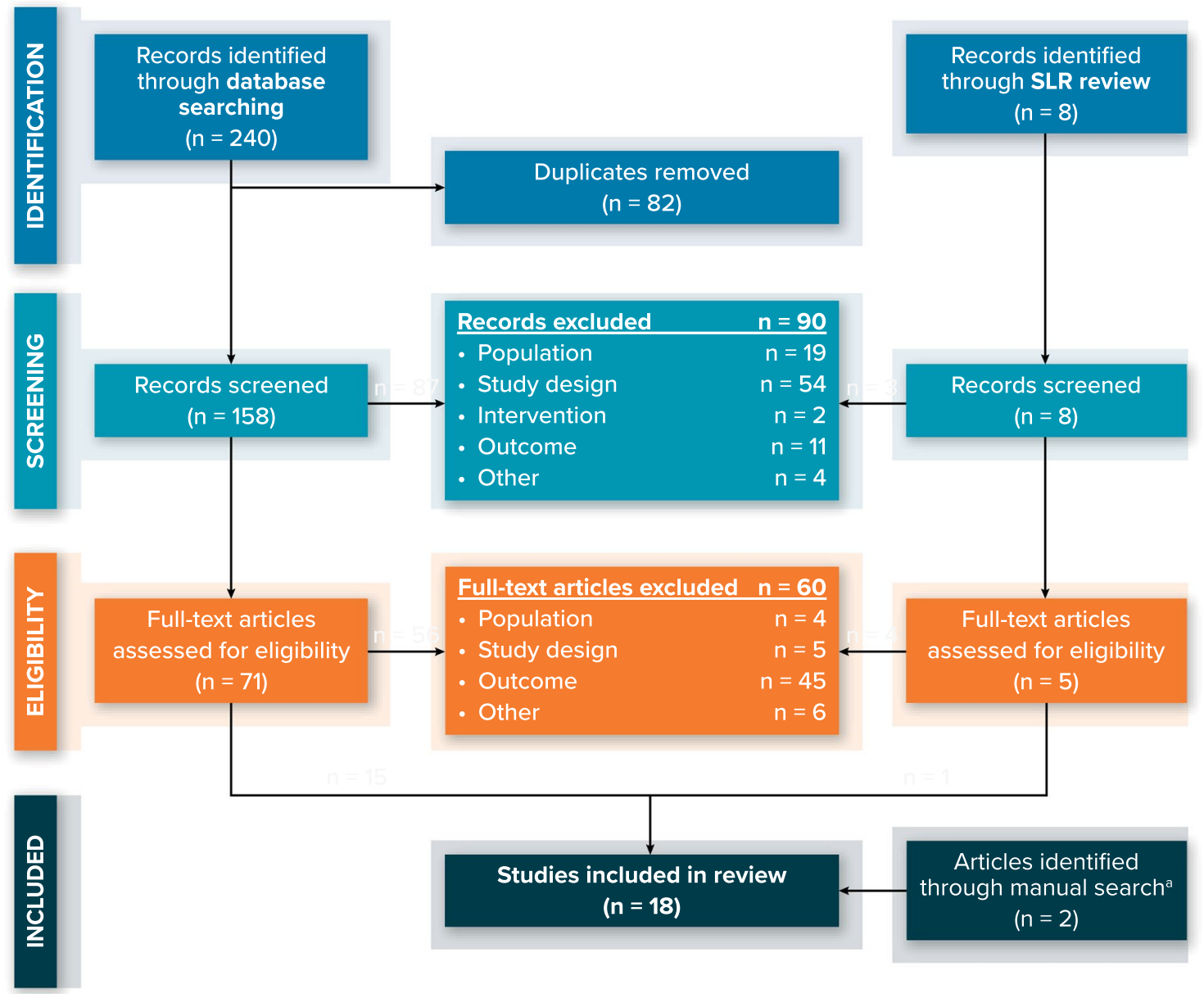

Fig. 1 PRISMA Diagram of Articles Included in Review. HER2 human epidermal growth factor receptor 2, PRISMA preferred reporting items for systematic reviews and meta-analyses, SLR systematic

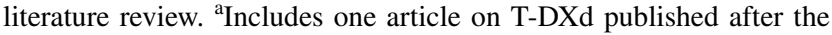

search cut-off date [15], and one phase 1 study describing trastuzumab duocarmazine, an investigational anti-HER2 drug that is suspected to be associated with treatment-related interstitial lung disease [16] lines (median) of systemic therapy for advanced or metastatic disease ranged from one line [19] to five or more lines of therapy $[15,21,27]$, with $2-4$ previous lines of therapy being the most commonly reported $[16,17,26$, 29-31].

Articles in this review reported on adverse drug reactions for currently approved or investigational anti-HER2 therapies. The approved therapies include trastuzumab combination therapy, T-DM1, T-DXd (approved in the US), and lapatinib combination therapy. Trastuzumab duocarmazine was the only investigational therapy. Patient enrollment was conducted during 2001-2017 for trastuzumab studies, up to 2008 for lapatinib studies, up to 2014 for T-DM1 studies, 2015-2018 for T-DXd studies, and 2014-2018 for the trastuzumab duocarmazine study.

\section{Drug-induced ILD incidence and severity}

A summary of drug-induced ILD incidence and severity reported in each study is presented in Table 2. A summary of drug-induced ILD incidence and severity by anti-HER2 therapy, including trastuzumab, lapatinib, T-DM1, T-DXd, and trastuzumab duocarmazine is provided in Table $\mathrm{S}-2$ (Supplementary Material).

\section{Trastuzumab}

Eight studies reported incidence and severity of druginduced ILD in a total of 1,642 patients receiving trastuzumab therapy; of these patients, $162(9.9 \%)$ had a reported ILD event. Overall, there were $3(0.2 \%)$ ILD-related deaths among those receiving trastuzumab therapy. Two 


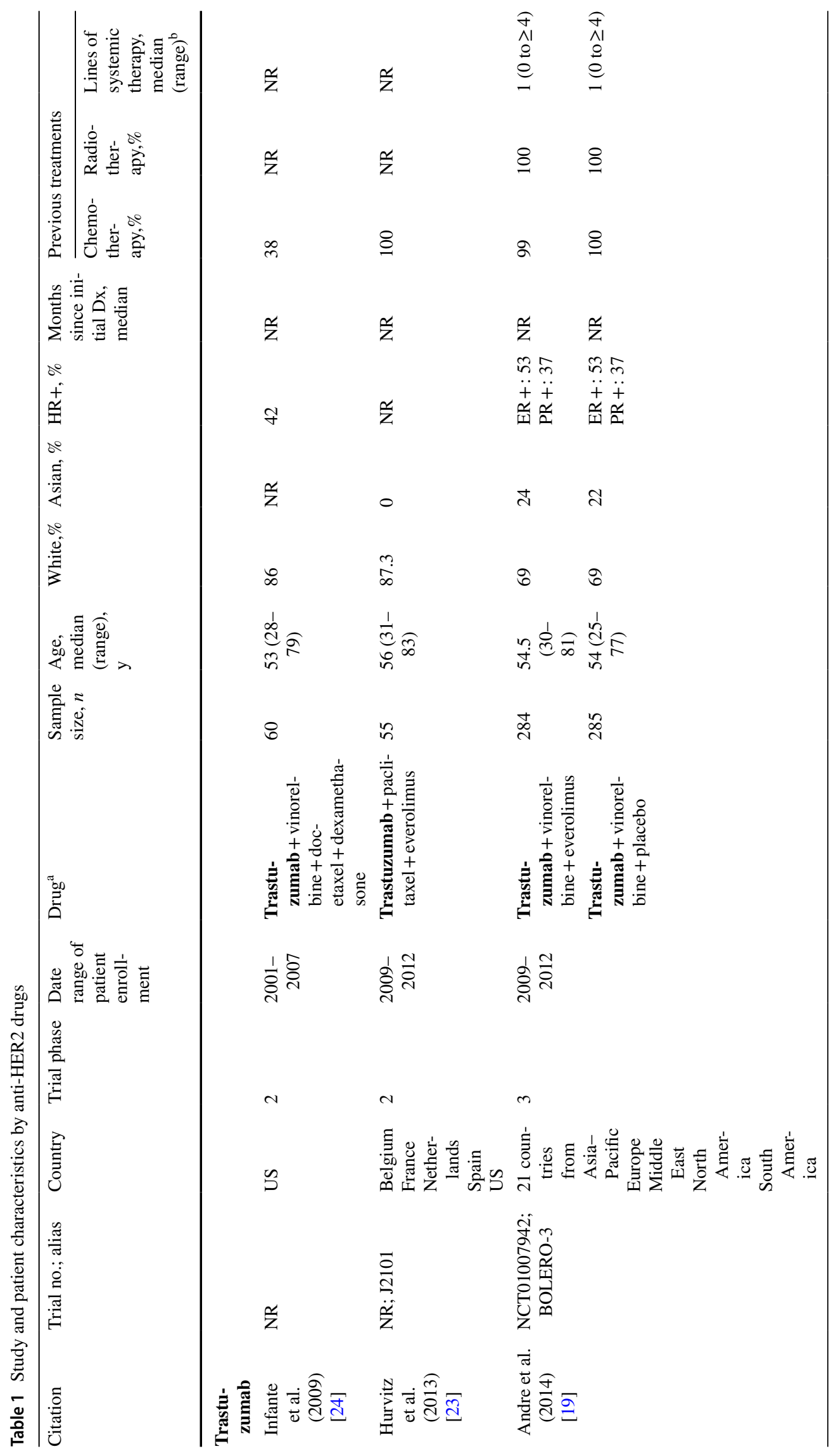




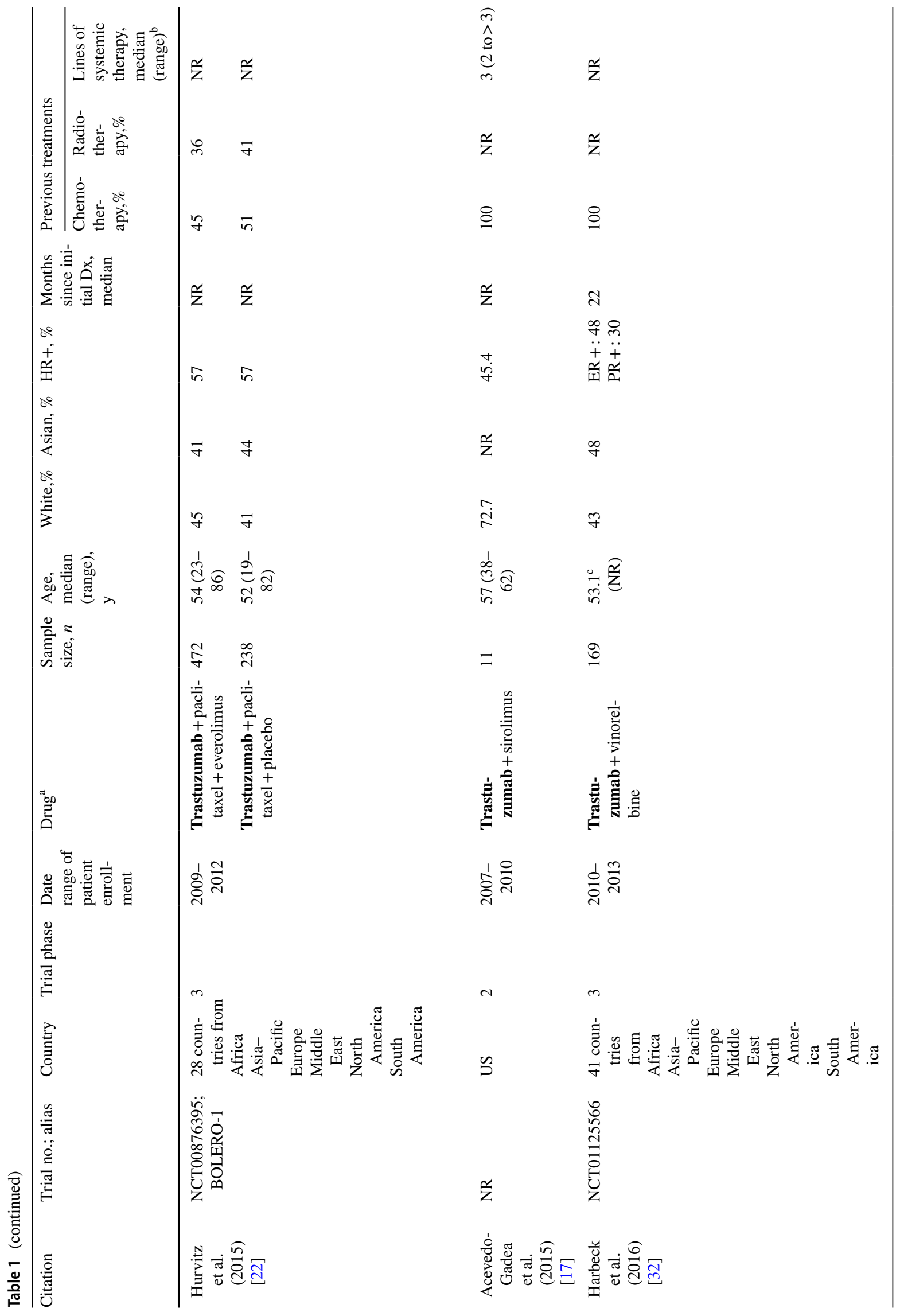




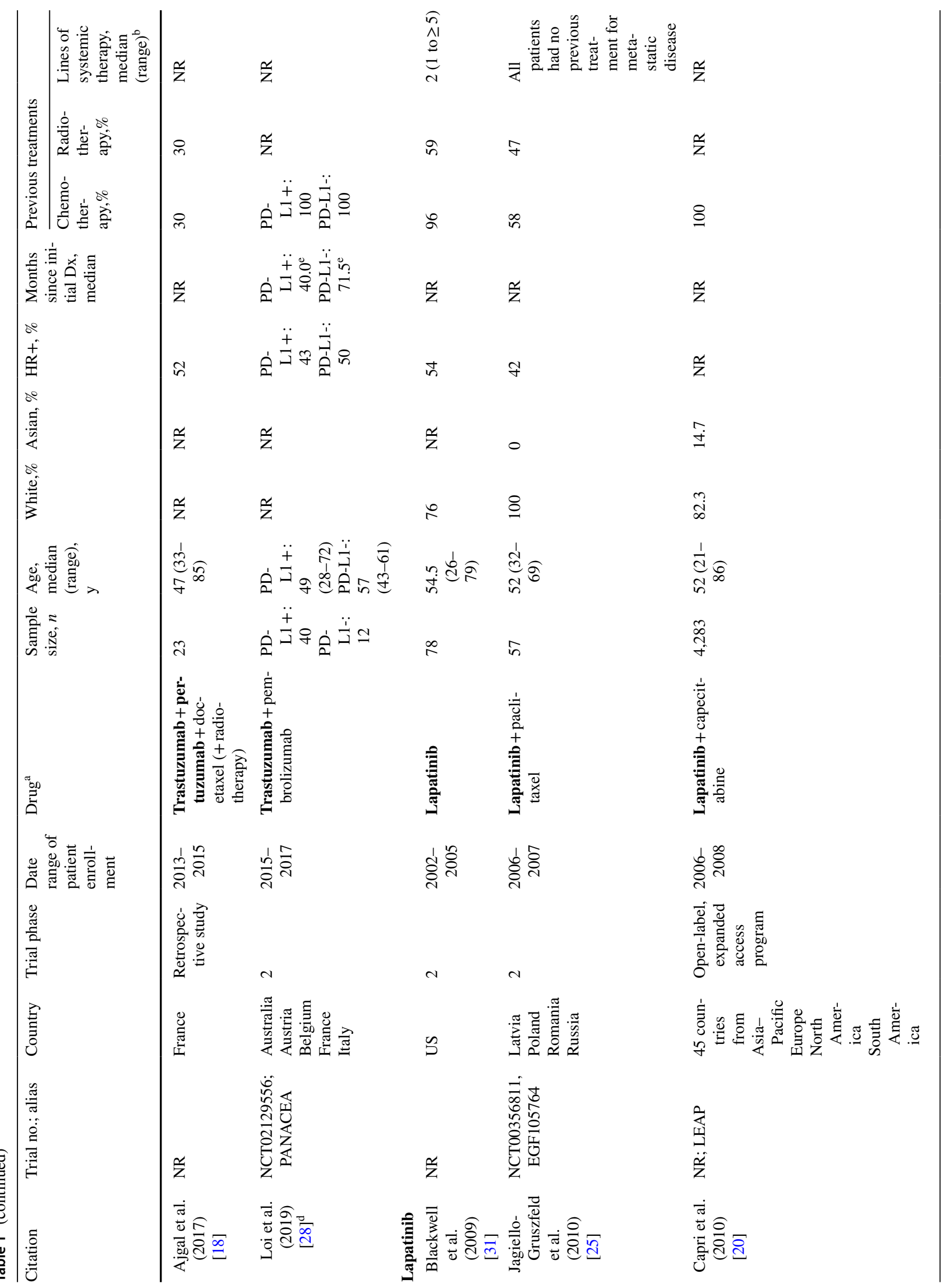




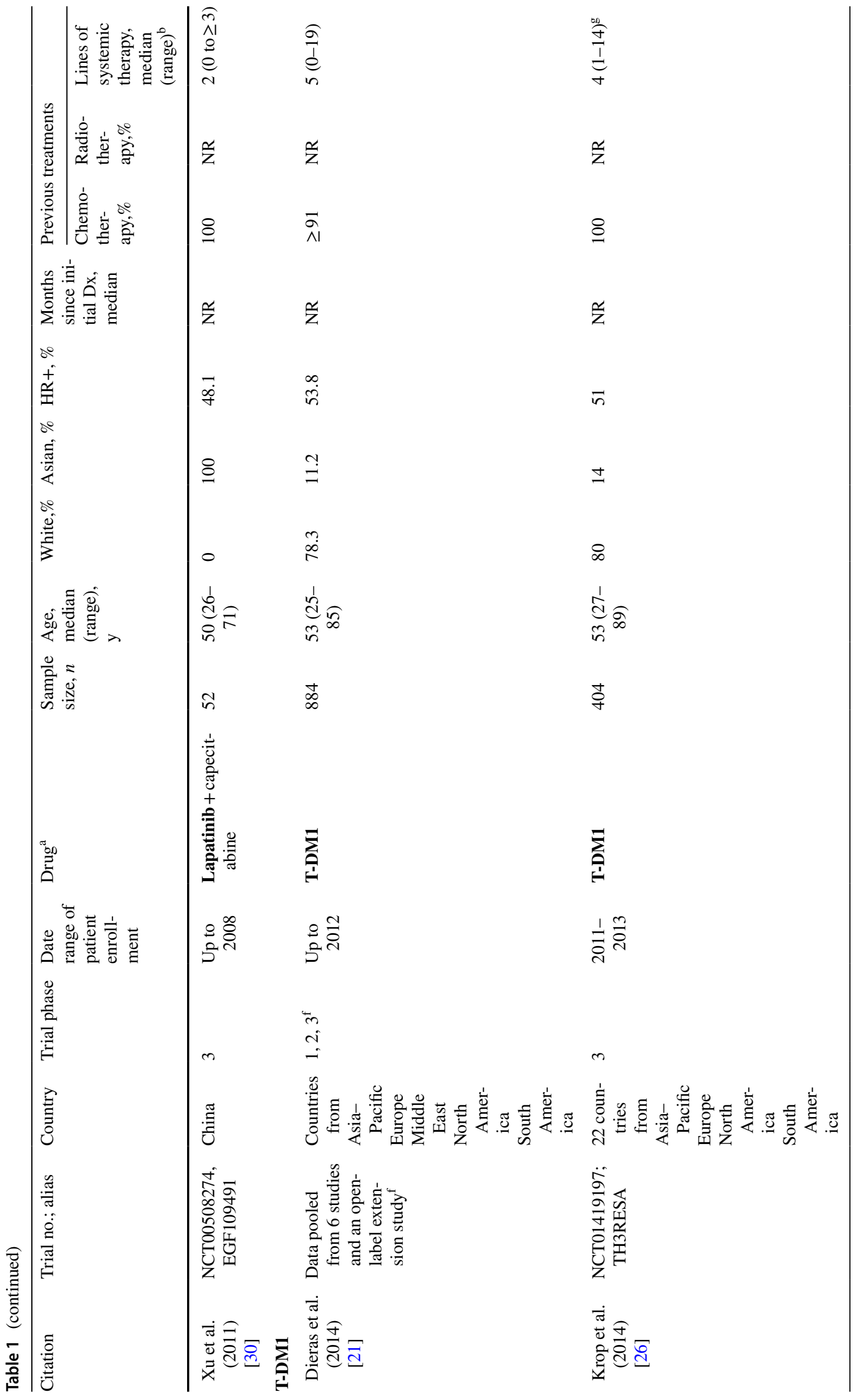




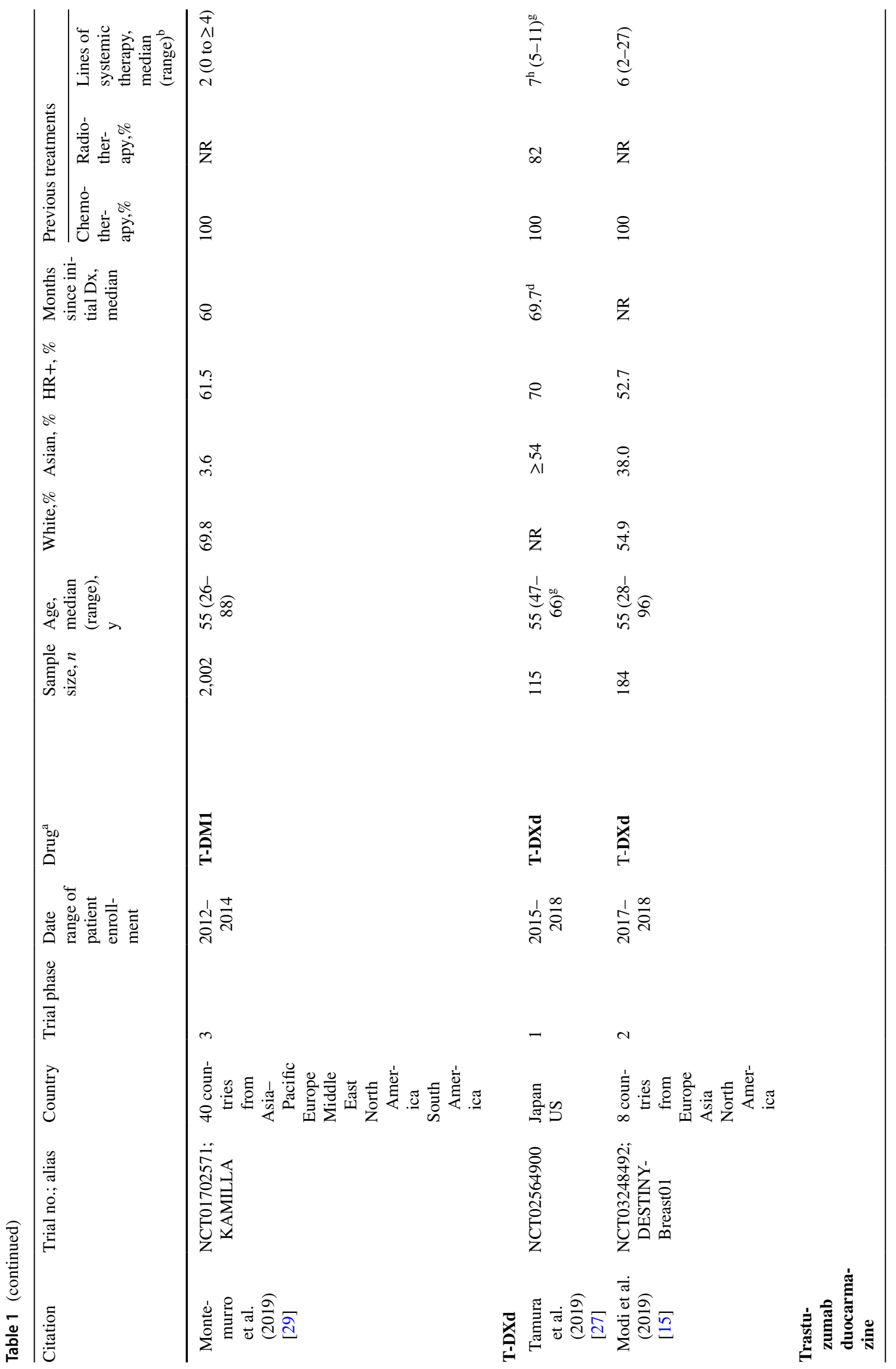




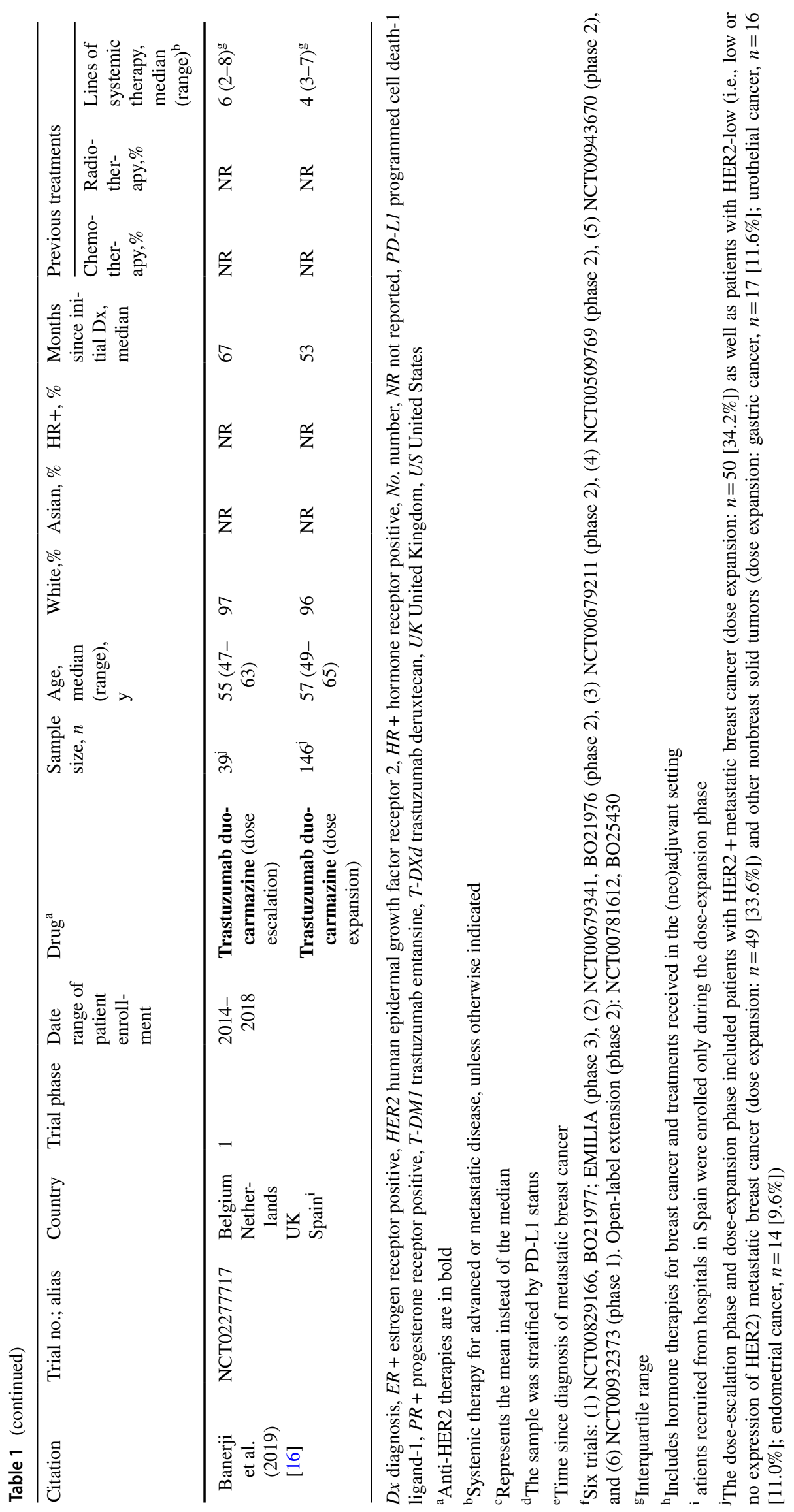




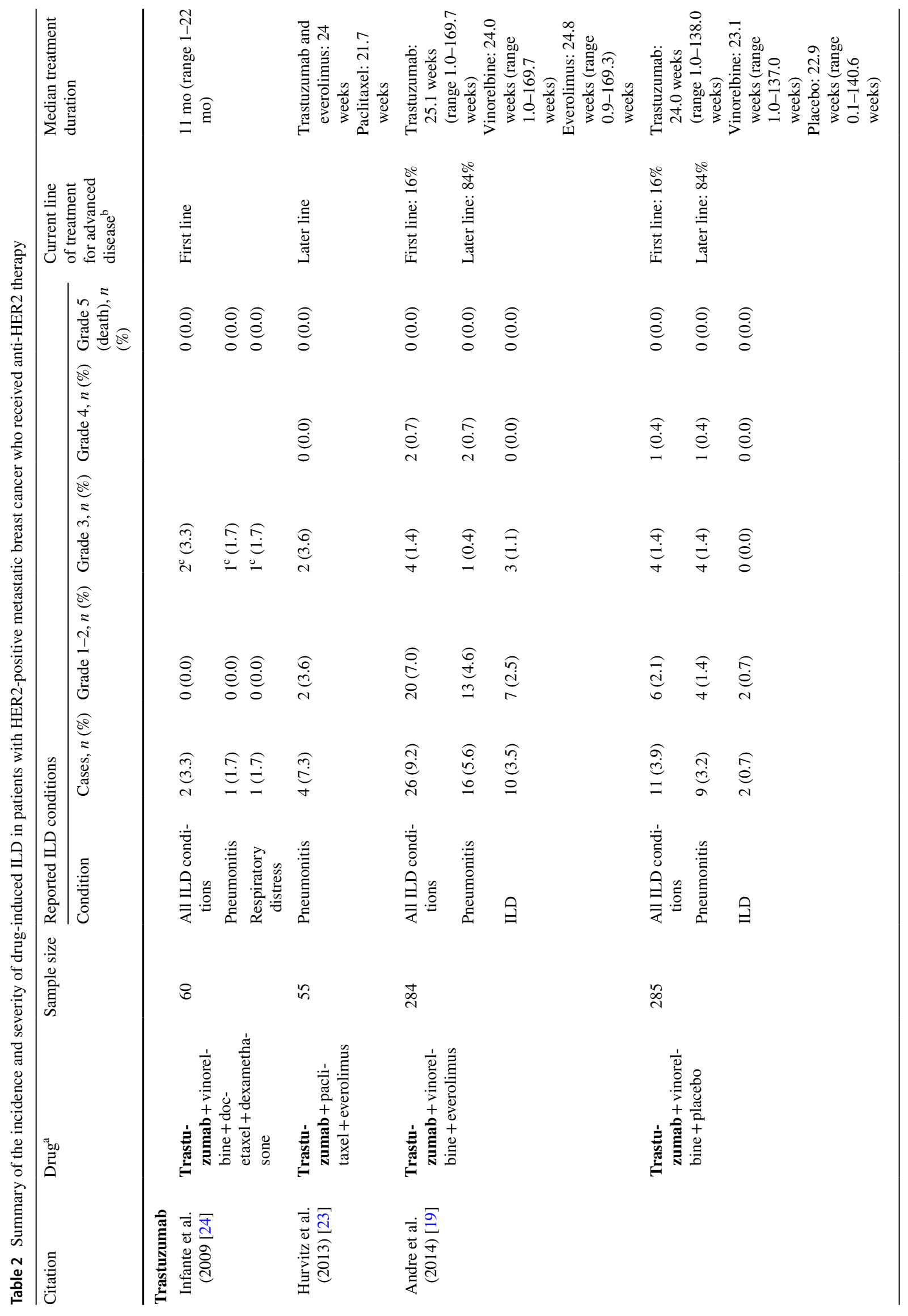




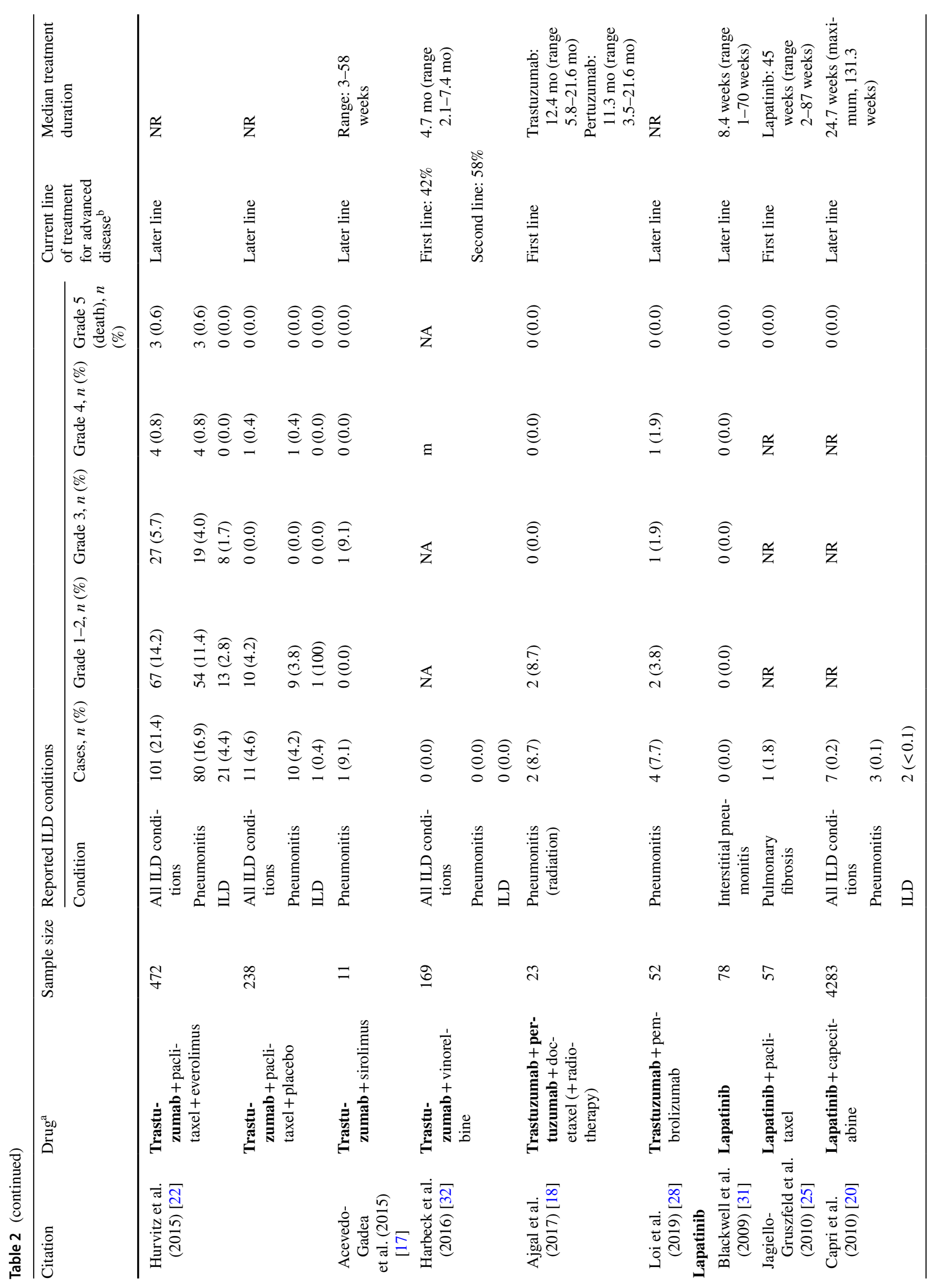




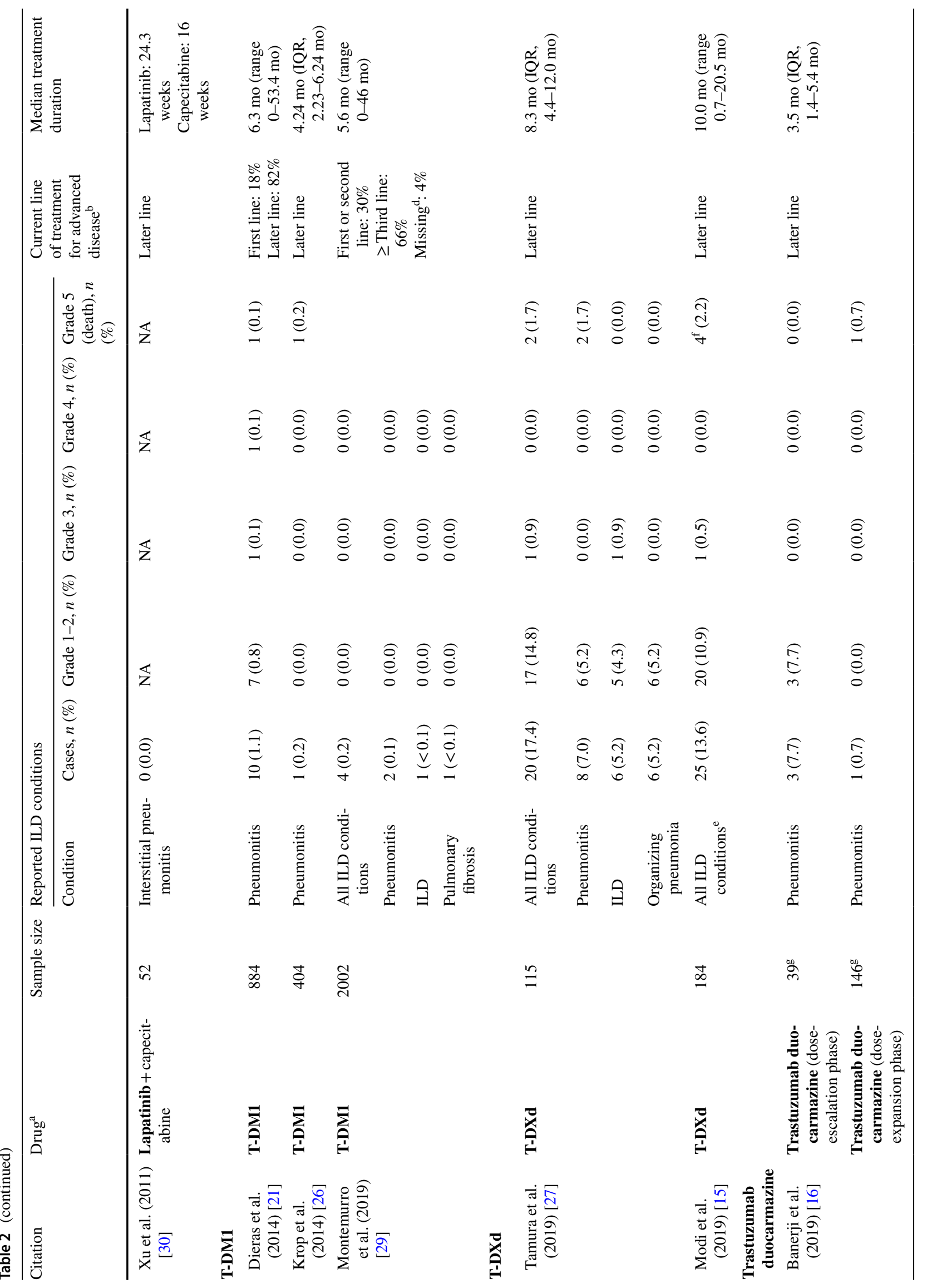


trastuzumab studies reported at least one case of specifically ILD [19, 22]. Seven trastuzumab studies reported at least one case of pneumonitis [17-19, 22-24, 28], of which six studies reported at least one case of grade 3 or higher pneumonitis and one study reported grade 1-2 pneumonitis cases related to radiotherapy [18]. Four studies assessed patients who received trastuzumab combined with an mTOR inhibitor, including everolimus [19, 22, 23] and sirolimus [17]. One study reported an ILD incidence of $9.2 \%(n=26)$ among patients who received trastuzumab for 25.1 weeks (median) combined with vinorelbine and everolimus; the ILD incidence was lower in the comparator $\operatorname{arm}(n=11$ [3.9\%]), which received trastuzumab for 24.0 weeks (median) combined with vinorelbine and placebo instead of everolimus; however, compared with the everolimus arm, a similar proportion of patients in the comparator arm $(n=5$ [1.8\%]) were diagnosed with grade 3 or 4 ILD [19]. Other studies with patients receiving trastuzumab combined with vinorelbine, either alone for 4.7 months (median) [32] or combined with docetaxel (a taxane chemotherapeutic agent) and dexamethasone (a corticosteroid) for 11 months (median) [24], reported a lower ILD incidence $(n=0$ [0\%] and $n=2$ [3.3\%], respectively) than studies with patients who received trastuzumab combined with an mTOR inhibitor.

\section{Lapatinib}

Four studies reported on a total of 4,470 patients who received lapatinib, a dual epidermal growth factor receptor/HER2 tyrosine kinase inhibitor; eight of these patients $(0.2 \%)$ had at least one reported ILD event. The earliest of these studies reported no ILD cases among patients who received lapatinib for 8.4 weeks (median) [31]. An expanded access study conducted in 45 countries (sample size, $n=4,283$ ) [20] and a phase 3 study conducted in China (sample size, $n=52$ ) [30] reported an ILD incidence of $0.2 \%$ ( $n=7$ ) and $0.0 \%$ (no cases), respectively, among patients who received lapatinib, combined with capecitabine, for 24.7 and 24.3 weeks (median), respectively. Among all studies that assessed lapatinib-based therapies, the highest ILD incidence ( $1.8 \%$, consisting of one case of pulmonary fibrosis) was reported in a phase 2 study (sample size, $n=57$ ) conducted in eastern Europe among patients who received lapatinib combined with paclitaxel for 45 weeks (median) [25].

\section{Trastuzumab emtansine}

Three studies reported on 3,290 patients who received T-DM1, an antibody-drug conjugate that contains the trastuzumab monoclonal antibody bound to the cytotoxic agent DM1, with a treatment duration ranging from 4.2 to 6.3 months (median) $[21,26,29]$. Across these studies, 15 
patients $(0.5 \%)$ had a reported ILD event, and 6 patients $(0.2 \%)$ had an ILD-related death. Dieras et al. (sample size, $n=884$ ) reported the highest ILD incidence (pneumonitis, $n=10[1.1 \%]$ ), including 2 patients $(0.2 \%)$ with grade $3-4$ pneumonitis and one pneumonitis-related death $(0.1 \%)$ [21]. Both Krop et al. (sample size, $n=404$ ) and Montemurro et al. (sample size, $n=2,002$ ) only reported deaths related to ILD, both with an incidence of $0.2 \%$ ( $n=1$ and $n=4$, respectively) [26, 29].

\section{Trastuzumab deruxtecan}

T-DXd is a HER2-targeted antibody-drug conjugate composed of a humanized monoclonal antibody with the same amino acid sequence as trastuzumab, a cleavable tetrapeptide-based linker, and a potent topoisomerase I inhibitor payload [33-35]. A phase 1 study enrolled 115 patients (doseexpansion phase) from Japan $(n=62[54 \%])$ and the US $(n=53$ [46\%]) who received T-DXd for 8.3 months (median) and reported an ILD incidence of $17.4 \%(n=20$; grade $1-2$, $n=17$ [incidence, $14.8 \%$ ]; grade $3, n=1$ [incidence, $0.9 \%$ ]; no grade 4 ; deaths, $n=2$ [incidence, $1.7 \%$ ]), including cases of pneumonitis $(n=8)$, specifically ILD $(n=6)$, and organizing pneumonia $(n=6)$ [27]. The phase 2 study assessed 184 patients enrolled from North America $(n=53$ [28.8\%]), Asia $(n=63[34.2 \%])$, and Europe $(n=n=68$ [37.0\%]) who received T-DXd for 10.0 months (median) [15]. Twenty-five patients (13.6\%) had a treatment-related ILD event, most of which were grade $1-2(n=20$ [incidence, $10.9 \%$ ]), one grade 3 (incidence, $0.5 \%$ ), no grade 4 , and four ILD-related deaths (incidence, $2.2 \%$ ).

\section{Trastuzumab duocarmazine}

Trastuzumab duocarmazine is a HER2-targeted antibody-drug conjugate composed of trastuzumab bound to a linker drug containing duocarmycin. One phase 1 study reported on ILD among patients with either MBC $(n=99$ [67.8\%] in the dose-expansion phase), gastric cancer, urothelial cancer, or endometrial cancer and receiving trastuzumab duocarmazine for 3.5 months (median) [16]. The study included a dose-escalation phase (sample size, $n=39$ ) and a dose-expansion phase (sample size, $n=146$ ) and reported three cases of pneumonitis (7.7\%, all grade 1-2) in the doseescalation phase and one pneumonitis-related death $(0.7 \%)$ in the dose-expansion phase.

\section{ILD severity by line of therapy for advanced disease}

Across all studies, the incidence of any-grade ILD was similar between patients who received an anti-HER2 drug as first-line therapy $(3.6 \%[n=5])$ versus later line therapy (2.3\% $[n=229])$ (Table S-3, Supplementary Material).
While studies reporting on patients who received an antiHER2 drug as first-line therapy reported no ILD-related deaths, studies assessing later line therapy reported a $0.2 \%$ incidence of ILD-related death $(n=16$, including ILD $[n=5(<0.1 \%)]$, pneumonitis $[n=10(0.1 \%)]$, and pulmonary fibrosis $[n=1(<0.1 \%)])$; however, among the studies included in this review, more patients received anti-HER2 therapy as later line therapy $(n=9,749)$ than as first-line therapy $(n=140)$.

Among patients who received an anti-HER2 drug as firstline therapy for advanced MBC, the ILD incidence was $1.8 \%$ $(n=1)$ [25], 3.3\% $(n=2)$ [24], and 8.7\% $(n=2)$ [18] across the three studies reporting on patients receiving lapatinibbased therapy for 10.4 months (median), trastuzumab-based therapy for 11 months (median), or trastuzumab-based therapy for 12.4 months (median) combined with pertuzumab for 11.3 months (median) and radiotherapy, respectively. In studies reporting only on patients who previously received systemic therapy for advanced MBC, the ILD incidence was as low as $0 \%$ among patients receiving lapatinib alone for 1.9 months (median) as later line therapy [31] and as high as $21.4 \%(n=101)$ among patients receiving trastuzumab combined with paclitaxel and everolimus as later line therapy (treatment duration not reported) [22]. Among patients who received T-DM1 for 4.2 months (median) as later line therapy, only one case $(0.2 \%)$ of pneumonitis was reported, which resulted in death [26]. Four studies reported on patients receiving either first-line or later line therapy for advanced or metastatic disease, with the majority of patients receiving later line therapy (range 58-84\%) across the studies $[19,21,29,32]$.

\section{Monitoring and management of ILD}

None of the studies in this review reported on specific guidelines for monitoring for ILD before an ILD diagnosis among patients receiving anti-HER2 therapies. However, 10 of the 18 studies $(55.6 \%)$ indicated that ILD-related events were managed via dose interruption, dose reduction, or treatment discontinuation [15-17, 22, 23, 25-28, 30]. The most common approaches included dose interruption and reduction for grades 2 and 3 and treatment discontinuation (often until the improvement of the ILD condition) for grades 3 and 4 .

Two studies in this review provided detailed guidelines for management of drug-induced ILD. In a phase 2 clinical trial of T-DXd, Modi et al. [15] recommend T-DXd dose interruption and possible systemic steroids for grade 1 events, and permanent T-DXd discontinuation with prompt initiation of systemic steroids for grade 2, 3, or 4 events; hospitalization is required for grade 3 or 4 events. In contrast, in a phase 3 clinical trial of trastuzumab combined with paclitaxel and either everolimus or placebo, Hurvitz et al. [22] recommend no specific treatment for grade 1 events, 
everolimus dose reduction and paclitaxel dose interruption for grade 2 events, additional dose interruption of everolimus and trastuzumab for grade 3 events, and treatment discontinuation for grade 4 events; corticosteroids are recommended for grades 2,3 , or 4 events.

\section{Discussion}

Drug-induced ILD has been reported among patients with HER2-positive MBC receiving anti-HER2 therapies, including trastuzumab, lapatinib, T-DM1, T-DXd, and trastuzumab duocarmazine. The highest ILD incidence was reported in patients who received trastuzumab combined with an mTOR inhibitor (everolimus [range 7.3-21.4\%] or sirolimus $[9.1 \%]$ ), and in patients who received T-DXd (range 13.6-17.4\%). The incidence of ILD-related deaths was highest among patients receiving T-DXd (range 1.7-2.2\%), whereas in patients receiving trastuzumab, lapatinib, T-DM1, or trastuzumab duocarmazine, the incidence was lower (range $0.1-0.6 \%$ ). The elevated incidence of druginduced ILD among those who received an mTOR inhibitor combined with trastuzumab is consistent with previous studies demonstrating associations between mTOR inhibitors and ILD among cancer patients [10, 36]. The lowest incidence of drug-induced ILD was among patients who received lapatinib-based therapy (range $0-1.8 \%$ ) or T-DM1 (range $0.2-1.1 \%$ ).

Disease management guidelines for drug-induced ILD were provided in two studies in this review: one on managing ILD induced by T-DXd [15] and the other on ILD induced by trastuzumab combined with paclitaxel and everolimus [22]. The guidance provided by these two studies differs, as they were conducted in the context of two different antiHER2 treatment regimens. The guidelines for trastuzumab in combination with paclitaxel and everolimus are consistent with the pneumonitis management guidance provided in the everolimus prescribing information [37], both recommending everolimus dose reduction and/or interruption for grade 2 or 3 events, but no change in treatment regimen for grade 1 . The guidelines for T-DXd-induced ILD recommend T-DXd dose interruption for grade 1 and permanent T-DXd treatment discontinuation for patients with grade 2,3 , or 4 , which is similar to the management recommendations for drug-induced lung injuries in Kubo et al. [13] and for pneumonitis induced by immune checkpoint inhibitors in the American Society of Clinical Oncology Practice Guideline [38]. The clinical significance of an ILD diagnosis together with the relatively high incidence of ILD induced by some anti-HER2 therapies highlights the need for consensus in ILD monitoring and management guidelines in the context of anticancer treatment for metastatic disease.
There are limitations associated with this review. The search was limited to the past 10 years and to English-language articles only. Additionally, studies reporting on ILD using a diagnosis term other than those prespecified in the inclusion criteria may have been excluded. Lastly, there was no quality assessment of the included studies (e.g., respiratory failure). However, a quality assessment of included studies was not necessary to accomplish the primary study objective of reporting on the incidence of ILD as an adverse drug reaction in the context of a clinical trial or observational study.

\section{Conclusions}

ILD is a well-described adverse drug reaction associated with anti-HER2 therapies, with the highest ILD incidence reported among patients receiving trastuzumab and everolimus combination therapy. Drug-induced ILD is typically managed via dose reduction, dose interruption, or treatment discontinuation; however, detailed ILD management guidelines are available for only two anti-HER2 treatment regimens (i.e., T-DXd and trastuzumab combined with paclitaxel and either everolimus or placebo), necessitating the development of standard guidelines across all anti-HER2 therapies for MBC. The dearth of published ILD monitoring approaches in the context of HER2-positive MBC and antiHER2 therapy highlights the need to identify risk factors and the underlying etiology of ILD to develop effective strategies for monitoring for ILD among these patients.

Acknowledgements The authors thank Brian Samsell of RTI Health Solutions for medical writing assistance.

Author contributions MDH, HED, JS, MER, and AL made substantial contributions to the conception or design of the work. All authors made substantial contributions to the acquisition, analysis, or interpretation of data for the work. All authors contributed to drafting the work or revising it critically for important intellectual content.

Funding Financial support for the study was provided by Daiichi Sankyo, Inc. RTI Health Solutions received funding under a research contract with Daiichi Sankyo, Inc. to conduct this study and provide editorial support in the form of manuscript writing, styling, and submission.

Data availability All data generated or analyzed during this study are included in this published article and its supplementary information files.

\section{Compliance with ethical standards}

Conflicts of interest MDH, JS, and CT are Daiichi Sankyo, Inc. employees. HED, AL, and MER are employees of RTI Health Solutions, an independent nonprofit research organization that performs contracted project work for medical device and pharmaceutical companies. DRC, HI, and CAP are consultants for Daiichi Sankyo, Inc. but have received no compensation for their contributions to this article. 
Ethics approval Not applicable for a literature review.

Informed consent Not applicable for a literature review.

Open Access This article is licensed under a Creative Commons Attribution 4.0 International License, which permits use, sharing, adaptation, distribution and reproduction in any medium or format, as long as you give appropriate credit to the original author(s) and the source, provide a link to the Creative Commons licence, and indicate if changes were made. The images or other third party material in this article are included in the article's Creative Commons licence, unless indicated otherwise in a credit line to the material. If material is not included in the article's Creative Commons licence and your intended use is not permitted by statutory regulation or exceeds the permitted use, you will need to obtain permission directly from the copyright holder. To view a copy of this licence, visit http://creativecommons.org/licenses/by/4.0/.

\section{References}

1. Howlader N, Altekruse SF, Li CI, Chen VW, Clarke CA, Ries LA, Cronin KA (2014) US incidence of breast cancer subtypes defined by joint hormone receptor and HER2 status. J Natl Cancer Inst. https://doi.org/10.1093/jnci/dju055

2. Goddard KA, Weinmann S, Richert-Boe K, Chen C, Bulkley J, Wax C (2012) HER2 evaluation and its impact on breast cancer treatment decisions. Public Health Genomics 15(1):1-10. https:// doi.org/10.1159/000325746

3. US Food and Drug Administration (2018) Herceptin prescribing information. https://www.accessdata.fda.gov/drugsatfda_docs/ label/2018/103792s5345lbl.pdf

4. US Food and Drug Administration (2019) Kadcyla prescribing information. https://www.accessdata.fda.gov/drugsatfda_docs/ label/2019/125427s105lbl.pdf

5. US Food and Drug Administration (2017) Nerlynx prescribing information. https://www.accessdata.fda.gov/drugsatfda_docs/ label/2017/208051s000lbl.pdf

6. US Food and Drug Administration (2018) Perjeta prescribing information. https://www.accessdata.fda.gov/drugsatfda_docs/ label/2018/125409s123lbl.pdf

7. US Food and Drug Administration (2018) Tykerb prescribing information. https://www.accessdata.fda.gov/drugsatfda_docs/ label/2018/022059s024lbl.pdf

8. Sears CR, Peikert T, Possick JD, Naidoo J, Nishino M, Patel SP, Camus P, Gaga M, Garon EB, Gould MK, Limper AH, Montgrain PR, Travis WD, Rivera MP (2019) Knowledge gaps and research priorities in immune checkpoint inhibitor-related pneumonitis: an official American Thoracic Society research statement. Am J Respir Crit Care Med 200(6):e31-e43. https://doi.org/10.1164/ rccm.201906-1202ST4

9. US Food and Drug Administration (2019) FDA warns about rare but severe lung inflammation with Ibrance, Kisqali, and Verzenio for breast cancer. https://www.fda.gov/drugs/drug-safety-and-avail ability/fda-warns-about-rare-severe-lung-inflammation-ibrancekisqali-and-verzenio-breast-cancer. Accessed October 15, 2019

10. Willemsen AE, Grutters JC, Gerritsen WR, van Erp NP, van Herpen CM, Tol J (2016) mTOR inhibitor-induced interstitial lung disease in cancer patients: comprehensive review and a practical management algorithm. Int J Cancer 138(10):2312-2321. https:// doi.org/10.1002/ijc. 29887

11. American Thoracic Society (2010) Interstitial lung disease. In: Schraufnagel DE (ed) Breathing in America: disease, progress, and hope
12. Meyer KC (2014) Diagnosis and management of interstitial lung disease. Transl Respir Med 2:4. https://doi. org/10.1186/2213-0802-2-4

13. Kubo K, Azuma A, Kanazawa M, Kameda H, Kusumoto M, Genma A, Saijo Y, Sakai F, Sugiyama Y, Tatsumi K, Dohi M, Tokuda H, Hashimoto S, Hattori N, Hanaoka M, Fukuda Y, Japanese Respiratory Society Committee for formulation of Consensus statement for the diagnosis, treatment of drug-induced lung injuries (2013) Consensus statement for the diagnosis and treatment of drug-induced lung injuries. Respir Investig 51(4):260-277. https ://doi.org/10.1016/j.resinv.2013.09.001

14. Wells AU, Hirani N (2008) Interstitial lung disease guideline. Tho$\operatorname{rax}$ 63(Suppl 5):v1-v58. https://doi.org/10.1136/thx.2008.101691

15. Modi S, Saura C, Yamashita T, Park YH, Kim SB, Tamura K, Andre F, Iwata H, Ito Y, Tsurutani J, Sohn J, Denduluri N, Perrin C, Aogi K, Tokunaga E, Im SA, Lee KS, Hurvitz SA, Cortes J, Lee C, Chen S, Zhang L, Shahidi J, Yver A, Krop I, Investigators DE-B (2019) Trastuzumab deruxtecan in previously treated HER2-positive breast cancer. New Engl J Med. https://doi. org/10.1056/NEJMoa1914510

16. Banerji U, van Herpen CML, Saura C, Thistlethwaite F, Lord S, Moreno V, Macpherson IR, Boni V, Rolfo C, de Vries EGE, Rottey S, Geenen J, Eskens F, Gil-Martin M, Mommers EC, Koper NP, Aftimos P (2019) Trastuzumab duocarmazine in locally advanced and metastatic solid tumours and HER2-expressing breast cancer: a phase 1 dose-escalation and dose-expansion study. Lancet Oncol 20(8):1124-1135. https://doi.org/10.1016/S1470-2045(19)30328 $-6$

17. Acevedo-Gadea C, Hatzis C, Chung G, Fishbach N, LezonGeyda K, Zelterman D, DiGiovanna MP, Harris L, Abu-Khalaf MM (2015) Sirolimus and trastuzumab combination therapy for HER2-positive metastatic breast cancer after progression on prior trastuzumab therapy. Breast Cancer Res Treat 150(1):157-167. https://doi.org/10.1007/s10549-015-3292-8

18. Ajgal Z, de Percin S, Dieras V, Pierga JY, Campana F, Fourquet A, Kirova YM (2017) Combination of radiotherapy and double blockade HER2 with pertuzumab and trastuzumab for HER2positive metastatic or locally recurrent unresectable and/or metastatic breast cancer: assessment of early toxicity. Cancer Radiother 21(2):114-118. https://doi.org/10.1016/j.canrad.2016.10.002

19. Andre F, O'Regan R, Ozguroglu M, Toi M, Xu B, Jerusalem G, Masuda N, Wilks S, Arena F, Isaacs C, Yap YS, Papai Z, Lang I, Armstrong A, Lerzo G, White M, Shen K, Litton J, Chen D, Zhang Y, Ali S, Taran T, Gianni L (2014) Everolimus for women with trastuzumab-resistant, HER2-positive, advanced breast cancer (BOLERO-3): a randomised, double-blind, placebo-controlled phase 3 trial. Lancet Oncol 15(6):580-591. https://doi. org/10.1016/S1470-2045(14)70138-X

20. Capri G, Chang J, Chen SC, Conte P, Cwiertka K, Jerusalem G, Jiang Z, Johnston S, Kaufman B, Link J, Ro J, Schutte J, Oliva C, Parikh R, Preston A, Rosenlund J, Selzer M, Zembryki D, De Placido S (2010) An open-label expanded access study of lapatinib and capecitabine in patients with HER2-overexpressing locally advanced or metastatic breast cancer. Ann Oncol 21(3):474-480. https://doi.org/10.1093/annonc/mdp373

21. Dieras V, Harbeck N, Budd GT, Greenson JK, Guardino AE, Samant M, Chernyukhin N, Smitt MC, Krop IE (2014) Trastuzumab emtansine in human epidermal growth factor receptor 2-positive metastatic breast cancer: an integrated safety analysis. J Clin Oncol 32(25):2750-2757. https://doi.org/10.1200/ JCO.2013.54.4999

22. Hurvitz SA, Andre F, Jiang Z, Shao Z, Mano MS, Neciosup SP, Tseng LM, Zhang Q, Shen K, Liu D, Dreosti LM, Burris HA, Toi M, Buyse ME, Cabaribere D, Lindsay MA, Rao S, Pacaud LB, Taran T, Slamon D (2015) Combination of everolimus with trastuzumab plus paclitaxel as first-line treatment for patients with 
HER2-positive advanced breast cancer (BOLERO-1): a phase 3 , randomised, double-blind, multicentre trial. Lancet Oncol 16(7):816-829. https://doi.org/10.1016/S1470-2045(15)00051-0

23. Hurvitz SA, Dalenc F, Campone M, O'Regan RM, Tjan-Heijnen VC, Gligorov J, Llombart A, Jhangiani H, Mirshahidi HR, TanChiu E, Miao S, El-Hashimy M, Lincy J, Taran T, Soria JC, Sahmoud T, Andre F (2013) A phase 2 study of everolimus combined with trastuzumab and paclitaxel in patients with HER2overexpressing advanced breast cancer that progressed during prior trastuzumab and taxane therapy. Breast Cancer Res Treat 141(3):437-446. https://doi.org/10.1007/s10549-013-2689-5

24. Infante JR, Yardley DA, Burris HA 3rd, Greco FA, Farley CP, Webb C, Spigel DR, Hainsworth JD (2009) Phase II trial of weekly docetaxel, vinorelbine, and trastuzumab in the firstline treatment of patients with HER2-positive metastatic breast cancer. Clin Breast Cancer 9(1):23-28. https://doi.org/10.3816/ CBC.2009.n.004

25. Jagiello-Gruszfeld A, Tjulandin S, Dobrovolskaya N, Manikhas A, Pienkowski T, DeSilvio M, Ridderheim M, Abbey R (2010) A single-arm phase II trial of first-line paclitaxel in combination with lapatinib in HER2-overexpressing metastatic breast cancer. Oncology 79(1-2):129-135. https://doi.org/10.1159/000318043

26. Krop IE, Kim SB, Gonzalez-Martin A, LoRusso PM, Ferrero JM, Smitt M, Yu R, Leung AC, Wildiers H, TRs Collaborators (2014) Trastuzumab emtansine versus treatment of physician's choice for pretreated HER2-positive advanced breast cancer (TH3RESA): a randomised, open-label, phase 3 trial. Lancet Oncol 15(7):689699. https://doi.org/10.1016/S1470-2045(14)70178-0

27. Tamura K, Tsurutani J, Takahashi S, Iwata H, Krop IE, Redfern C, Sagara Y, Doi T, Park H, Murthy RK, Redman RA, Jikoh T, Lee C, Sugihara M, Shahidi J, Yver A, Modi S (2019) Trastuzumab deruxtecan (DS-8201a) in patients with advanced HER2-positive breast cancer previously treated with trastuzumab emtansine: a dose-expansion, phase 1 study. Lancet Oncol 20(6):816-826. https ://doi.org/10.1016/S1470-2045(19)30097-X

28. Loi S, Giobbie-Hurder A, Gombos A, Bachelot T, Hui R, Curigliano G, Campone M, Biganzoli L, Bonnefoi H, Jerusalem G, Bartsch R, Rabaglio-Poretti M, Kammler R, Maibach R, Smyth MJ, Di Leo A, Colleoni M, Viale G, Regan MM, Andre F, International Breast Cancer Study G, the Breast International G (2019) Pembrolizumab plus trastuzumab in trastuzumab-resistant, advanced, HER2-positive breast cancer (PANACEA): a singlearm, multicentre, phase 1b-2 trial. Lancet Oncol 20(3):371-382. https://doi.org/10.1016/S1470-2045(18)30812-X

29. Montemurro F, Ellis P, Anton A, Wuerstlein R, Delaloge S, Bonneterre J, Quenel-Tueux N, Linn SC, Irahara N, Donica M, Lindegger N, Barrios CH (2019) Safety of trastuzumab emtansine (T-DM1) in patients with HER2-positive advanced breast cancer: primary results from the KAMILLA study cohort 1 . Eur J Cancer 109:92-102. https://doi.org/10.1016/j.ejca.2018.12.022

30. Xu BH, Jiang ZF, Chua D, Shao ZM, Luo RC, Wang XJ, Liu DG, Yeo W, Yu SY, Newstat B, Preston A, Martin AM, Chi HD, Wang L (2011) Lapatinib plus capecitabine in treating HER2-positive advanced breast cancer: efficacy, safety, and biomarker results from Chinese patients. Chin J Cancer 30(5):327-335

31. Blackwell KL, Pegram MD, Tan-Chiu E, Schwartzberg LS, Arbushites MC, Maltzman JD, Forster JK, Rubin SD, Stein SH, Burstein HJ (2009) Single-agent lapatinib for HER2-overexpressing advanced or metastatic breast cancer that progressed on first- or second-line trastuzumab-containing regimens. Ann Oncol 20(6):1026-1031. https://doi.org/10.1093/annonc/mdn759

32. Harbeck N, Huang CS, Hurvitz S, Yeh DC, Shao Z, Im SA, Jung KH, Shen K, Ro J, Jassem J, Zhang Q, Im YH, Wojtukiewicz M, Sun Q, Chen SC, Goeldner RG, Uttenreuther-Fischer M, Xu B, Piccart-Gebhart M, LU-Bs Group (2016) Afatinib plus vinorelbine versus trastuzumab plus vinorelbine in patients with HER2overexpressing metastatic breast cancer who had progressed on one previous trastuzumab treatment (LUX-Breast 1): an openlabel, randomised, phase 3 trial. Lancet Oncol 17(3):357-366. https://doi.org/10.1016/S1470-2045(15)00540-9

33. Ogitani Y, Hagihara K, Oitate M, Naito H, Agatsuma T (2016) Bystander killing effect of DS-8201a, a novel anti-human epidermal growth factor receptor 2 antibody-drug conjugate, in tumors with human epidermal growth factor receptor 2 heterogeneity. Cancer Sci 107(7):1039-1046. https://doi.org/10.1111/cas.12966

34. Nakada T, Sugihara K, Jikoh T, Abe Y, Agatsuma T (2019) The latest research and development into the antibody-drug conjugate, [fam-] trastuzumab deruxtecan (DS-8201a), for HER2 cancer therapy. Chem Pharm Bull 67(3):173-185. https://doi. org/10.1248/cpb.c18-00744

35. Ogitani Y, Aida T, Hagihara K, Yamaguchi J, Ishii C, Harada N, Soma M, Okamoto H, Oitate M, Arakawa S, Hirai T, Atsumi R, Nakada T, Hayakawa I, Abe Y, Agatsuma T (2016) DS-8201a, a novel HER2-Targeting ADC with a novel DNA topoisomerase i inhibitor, demonstrates a promising antitumor efficacy with differentiation from T-DM1. Clin Cancer Res 22(20):5097-5108. https://doi.org/10.1158/1078-0432.ccr-15-2822

36. Skeoch S, Weatherley N, Swift AJ, Oldroyd A, Johns C, Hayton C, Giollo A, Wild JM, Waterton JC, Buch M, Linton K, Bruce IN, Leonard C, Bianchi S, Chaudhuri N (2018) Drug-induced interstitial lung disease: a systematic review. J Clin Med. https:// doi.org/10.3390/jcm7100356

37. Afinitor prescribing information (2018) https://www.accessdata .fda.gov/drugsatfda_docs/label/2018/022334s040,203985s013lbl. pdf

38. Brahmer JR, Lacchetti C, Schneider BJ, Atkins MB, Brassil KJ, Caterino JM, Chau I, Ernstoff MS, Gardner JM, Ginex P, Hallmeyer S, Holter Chakrabarty J, Leighl NB, Mammen JS, McDermott DF, Naing A, Nastoupil LJ, Phillips T, Porter LD, Puzanov I, Reichner CA, Santomasso BD, Seigel C, Spira A, Suarez-Almazor ME, Wang Y, Weber JS, Wolchok JD, Thompson JA, National Comprehensive Cancer N (2018) Management of immune-related adverse events in patients treated with immune checkpoint inhibitor therapy: American Society of Clinical Oncology Clinical Practice Guideline. J Clin Oncol 36(17):1714-1768. https://doi.org/10.1200/JCO.2017.77.6385

39. ICH (1994) Clinical safety data management: definitions and standards for expedited reporting E2A. https://www.ich.org/produ cts/guidelines/efficacy/efficacy-single/article/clinical-safety-datamanagement-definitions-and-standards-for-expedited-reporting. html

40. NCI (2017) Common terminology criteria for adverse events (CTCAE), version 5.0. https://ctep.cancer.gov/protocoldevelop ment/electronic_applications/docs/CTCAE_v5_Quick_Refer ence_8.5x11.pdf

Publisher's Note Springer Nature remains neutral with regard to jurisdictional claims in published maps and institutional affiliations. 